\title{
Clinical and radiographic characteristics of the chronic apical periodontitis in senegalese population
}

\author{
Seydina Ousmane Niang ${ }^{1,}$, , Anta Seck ${ }^{2}$, Diouma Ndiaye ${ }^{3}$, Florent K Kouakou ${ }^{4}$, Babacar Toure ${ }^{5}$ \\ ${ }^{1-3}$ Assistant Professor, ${ }^{5}$ Professor, ${ }^{1-3,5}$ Dept. of Conservative Dentistry and Endodontics, Cheikh Anta Diop University, Dakar BP: \\ 5005 Dakar, Senegal, Lecturer, ${ }^{4}$ Dept of Conservative Dentistry and Endodontics, UFR d'Odonto-Stomatologie, UFR d'Odonto- \\ Stomatologie, University Félix Houphouét Boigny, Abidjan, 22 BP: 612 Abidjan 22
}

*Corresponding Author:

Email: seydiniang@yahoo.fr

\begin{abstract}
Aim: This study proposes to describe the epidemiological, clinical and radiographic characteristics of Chronic Apical Periodontitis (CAP) in a population of patients attending Conservative Dentistry clinic of Odontology Department of University Cheikh Anta Diop of Dakar.

Methodology: This was an observational, descriptive, cross-sectional clinical study. Cases were included after clinical and radiographic diagnosis of a CAP.

The data collection was done form the following headings: (i) epidemiological data on socio-demographic characteristics (ii) the clinical data (ie reason of consultation, type of tooth, degree of mobility, integrity of periodontal attachment (periodontal probing), and the presence or absence of swelling or fistula); and (iii) radiographic data on ligament thickening and periapical status.

Results: 51 cases of chronic apical periodontitis were included in the study. The results showed male predominance (68.6\%) with a mean age of 31.1 years +/- 12,1. Clinical symptoms regarding tooth mobility, loss of periodontal attachment, and swelling were timid or even inconsistent. The an tero-superior sector was the most concerned, with a strong presence of the 5 and 4 peri apical index (PAI) scores with respective percentages of $45.1 \%$ and $31.4 \%$.

Conclusion: During the study, it should be noted that PAC are more common in men and have modest clinical signs; which shows that their diagnosis is mainly radiographic.
\end{abstract}

Keywords: Chronic apical periodontitis, Clinic, Radiography, Dakar.

\section{Introduction}

Chronic apical periodontitis are peri-radicular inflammatory lesions of endodontic origin (L.I.P.O.E.). This definition highlights its endodontic infectious origin, the inflammatory nature of the lesion and its periodontal situation.

They represent a major public health problem that is largely underestimated in many countries, as they have many features and clinical forms that are often overlooked by practitioners.

The prevalence of AP varies from $15 \%$ to $75 \%$ depending on the countries and populations studied around the world. In Senegal, prevalence studies indicate that $56.1 \%$ of endodontically treated teeth have an endodontic peri-radicular inflammatory lesion. ${ }^{1}$

The management of apical periodontitis consists of endodontic etiological treatment, which will restore the integrity of the periodontal attachment after reduction of the bacterial load under a suitable threshold. ${ }^{2}$

This management involves a diagnostic procedure including knowledge of the clinical and radiographic features of chronic apical periodontitis. ${ }^{3}$

The diagnosis of this pathology is essentially radiological, however it presents several clinical forms whose characteristics are often wrongly relegated to the background. These constitute the basis of the differential diagnosis with other peri-radicular pathologies having the same radiographic expression.
Thus, this present study proposes to describe the epidemiological, clinical and radiographic characteristics of Chronic Apical Periodontitis in a population of patients who came to consult at the endodontic clinic of the Department of Odontology of Dakar.

\section{Methodology}

This is an observational clinical study describing the epidemiological, clinical and radiological characteristics of chronic apical periodontitis.

Study population and selections criteria: The cases were selected from the regular pool of patients attending Conservative Dentistry clinic of Odontology Department of Dakar after a clinical and radiographic diagnosis evoking a chronic apical periodontitis.

Included in this study were patients who met the following criteria: (i) patients aged over 16 years, (ii) clinical and radiological diagnosis of chronic apical periodontitis, (iii) informed consent signed by the patient.

The criteria for non-inclusion were: (i) patient with restraint preventing objective assessment of tooth mobility, (ii) pregnant patient (in case of need of several radios) (iii) periodontal involvement in the lesion (periodontal lesion mimicking endodontic lesion or true endo-periodontal lesion). 
Collection process and studied variables: The collection of data for this study was based on an index form of collection prepared on the basis of that used for the clinical teaching of students.

This collection sheet contains the following sections: epidemiological data; clinical data; radiographic data.

Regarding the epidemiological data, sociodemographic characteristics relating to age, sex and socio-economic level were recorded.

For clinical data, the following information was collected: the reason of consultation; the type of tooth; the degree of mobility. the integrity of the periodontal attachment (periodontal probing), and the presence or absence of swelling or fistula.

The classification of MULEHMAN (4) was used as a basis for evaluating tooth mobility with the different stages, namely. (i) physiological mobility. (ii) mobility less than $1 \mathrm{~mm}$ in the vestibulo-lingual direction. (iii) mobility greater than $1 \mathrm{~mm}$ but without alternation of the patient's function; (iv) vertical displacement and disturbed function.

Regarding radiographic data, the following characteristics were noted: the presence or absence of ligament thickening and periapical status.

With regard to periapical status, the periapical index (Periapical index of Ørstavik) (5) was used as a basis for evaluation with its five stages of evolution ie: (i) Structure periapical normal, (ii) small change in bone structure, (iii) structural alteration and mineral loss, (iv) apical periodontitis with well-defined clear radiographic image, (v) severe apical periodontitis with exacerbation.

Statistical Analyses: Collection and analysis of the data were carried out with SPSS (version 18.0). The qualitative variables were expressed as percentages, and the quantitative variables as a mean $\pm(\mathrm{SD})$ standard deviation.

Differences between qualitative variable were analyzed with the $\mathrm{Chi}^{2}$ test. The Mann-Whitney U and chi-square test was used to analyze the association between the variables. The significance level was fixed at $5 \%(\mathrm{p} \leq 0$, $05)$.

\section{Results}

Epidemiological characteristics: A total of 51 patients aged 17-66 years, were recruited. The average age was 31.1 years with a standard deviation. ${ }^{12,1}$ The most representative age groups are those between 26 and 45 years followed by that between 17 and 25 years, this difference is statistically significant P-value $=0.005$. (Table 1).

The distribution of the population by gender shows a predominance of men $(68.6 \%)$ compared to women $(31.4 \%)$ with a sex ratio (male / female) of 2.19 with a statistically significant difference $\mathrm{P}-$ Value $=0.008$ (Fig. 1).
The distribution of the population according socioeconomic status showed a strong predominance of students, followed by independent professionals, with a low frequency of retirees and unemployed peoples. The difference is statistically significant P-Value $=0.001$ (Table 2).

\section{Clinical Characteristics}

Reasons for consultation: The majority of patients (86.3\%) came for consultation for aesthetic and / or functional reasons and only 7 patients (13.7\%) for a systematic visit, with a statistically significant difference P-Value $=0.001$ (Fig. 2).

Type of Tooth: The distribution of the population according tooth type showed that the anterior maxillary area, especially the incisors, was the most concerned with a percentage of $68.6 \%(n=35)$, followed by molars with $23.5 \%$, and finally premolars with $7.8 \%$, with a statistically significant difference P-Value = 0.001. (Table 3)

Periodontal Probing - Sinus tract-Swelling: The evaluation of the integrity of the periodontal attachment showed that only three cases $(5.6 \%)$ presented a probing depths.

Clinical observation showed only one case (2\%) with a sinus tract in the vestibule opposite the causal tooth.

Mucosal examination showed no swelling in the vast majority of cases $(\mathrm{n}=49,96.1 \%)$ and only 2 cases (3.9\%) had a slight peripapical swelling.

Degree of mobility of the causal tooth: The assessment of the degree of mobility of the tooth according to the Mühlemann classification showed that the majority of cases $(\mathrm{N}=45$ or $88.2 \%)$ had a physiological mobility, and that few cases showed visible but inferior transversal mobility at $1 \mathrm{~mm}$ and others greater than $1 \mathrm{~mm}$ each with 3 cases (ie 5.9\%) with a statistically significant difference because PValue $=0.001($ Table 4$)$.

\section{Radiological Characteristics}

Thickening ligament: Radiographic assessment of the periodontal status showed ligament thickening in $82.4 \%$ of cases (42 cases) (Fig. 3).

Peri apical status: The evaluation of the peri apical status showed that the scores 5 and 4 of the peri apical index (PAI) are the most recorded with respectively $45.1 \%$ and $31.4 \%$. The low scores 3 and 2 are the least encountered in the present study with respectively $15.7 \%$ and $7.8 \%$. The difference is statistically significant $\mathrm{P}-$ Value $=0.001$ (Fig. 4). 


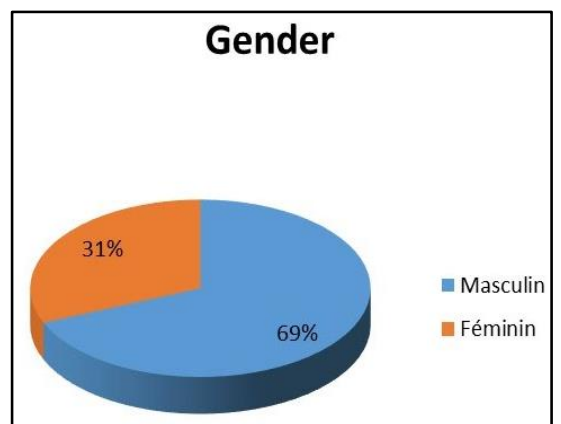

Fig. 1: Population distribution by gender

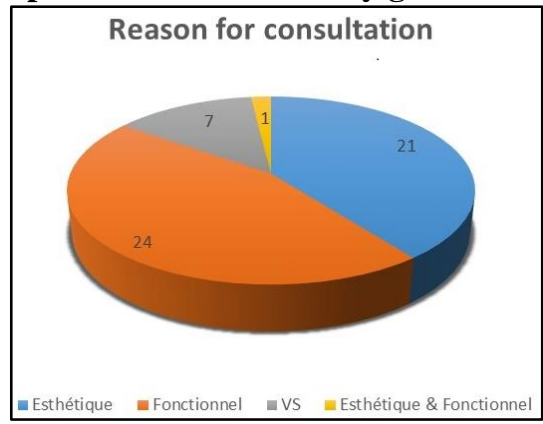

Fig. 2: Population distribution according the reason for consultation

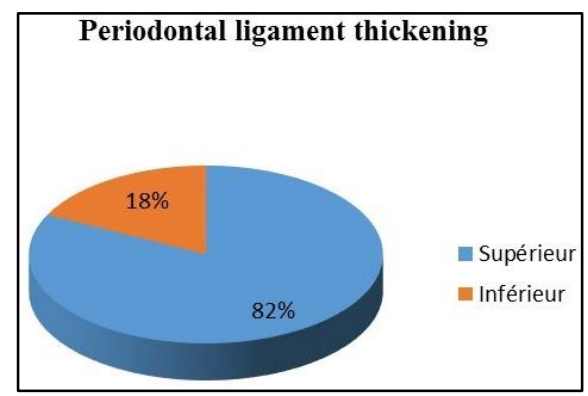

Fig. 3: Teeth distribution according periodontal ligament thickening

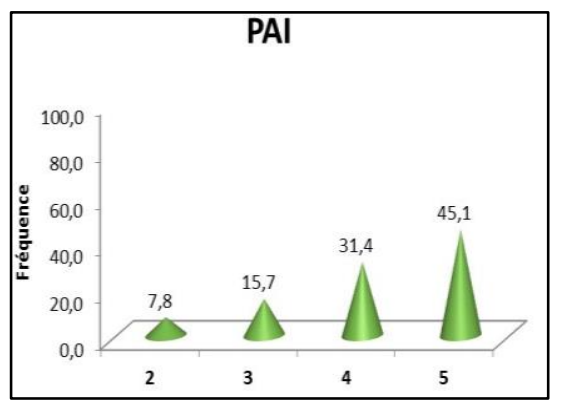

Fig. 4: Teeth distribution according peri apical status

Table 1: Population distribution by age and percentage

\begin{tabular}{|l|l|l|}
\hline Age Groups & Number (n) & Fréquence (\%) \\
\hline$[17-25]$ & 22 & 43,1 \\
\hline$[26-45]$ & 23 & 45,1 \\
\hline$[46-66]$ & 6 & 11,8 \\
\hline Total & 51 & 100,0 \\
\hline
\end{tabular}

Table 2: Population distribution according socioeconomic status

\begin{tabular}{|l|c|c|}
\hline Profession & Number(n) & Fréquence(\%) \\
\hline Informal sector & 11 & 21,6 \\
\hline $\begin{array}{l}\text { Independent } \\
\text { professionals }\end{array}$ & 12 & 23,5 \\
\hline Students & 23 & 45,1 \\
\hline Retirees & 2 & 3,9 \\
\hline Unemployed & 3 & 5,9 \\
\hline Total & 51 & 100,0 \\
\hline
\end{tabular}

Table 3: Population distribution according tooth type

\begin{tabular}{|c|c|c|}
\hline Tooth type & Number (n) & Fréquence (\%) \\
\hline I & 35 & 68,6 \\
\hline PM & 4 & 7,8 \\
\hline M & 12 & 23,5 \\
\hline Total & 51 & 100,0 \\
\hline
\end{tabular}

Table 4: Teeth distribution according degree of mobility

\begin{tabular}{|c|c|c|}
\hline Mobility & Number (n) & Fréquence (\%) \\
\hline 1 & 45 & 88,2 \\
\hline 2 & 3 & 5,9 \\
\hline 3 & 3 & 5,9 \\
\hline Total & 51 & 100,0 \\
\hline
\end{tabular}

\section{Discussion}

The appearance of apical periodontitis is based today on well-known etiological and pathological mechanisms. It is an inflammatory process localized in the peri-radicular region (usually periapical) in response to endodontic aggression.

Because of this anatomical particularity, the main limitation of this work is the absence of an at omopathological examination to characterize the histological nature of the lesions, and thus to differentiate the different pathological entities from the chronic apical periodontitis included in the study.

However, standardized and widely documented clinical and radiographic parameters in the scientific literature were used as a basis for evaluation of the study.

The anatomical and etiopathogenic characteristic of apical periodontitis is often accompanied by a destruction of the periodontal attachment. Thus, to characterize the degree of tooth mobility, the Mühlemann classification was the reference element in the present study because it is widely used in epidemiological and clinical studies, especially in Periodontology. ${ }^{6}$

In this study, retro-alveolar radiographs were used for peri apical status assessment, a technique also used by several authors. ${ }^{7,8}$

The periapical index used to evaluate the periapical status of teeth has been described since 1986 by 
Orstavik $^{5}$ for retro-alveolar radiographs. To avoid interevaluator variability, the latter were previously calibrated to the PAI system. X-ray images were also analyzed under the same conditions and by the same calibrated evaluators, different from the principal investigator.

However, with the development of 3D imaging such as the Cone Beam CBCT, has more finding, objectifies and quantitative analysis of peri-radicular bone loss and widening of periodontal space could been achieved. ${ }^{9,10}$

Sociodemographically, male patients with a sex ratio of 2.19. The average age was 31.1 years $+/-12.4$. The most representative age groups are those between 26 and 45 years of age, followed by those between 17 and 25 years old.

The predominance of men could be explained by the fact that the majority of teeth included in the present study are PAC secondary to an old dental trauma and whose male sex is generally the most exposed. Indeed, gender is a risk factor for extrinsic alveolo dental injuries. ${ }^{11}$

In addition, this age group is the favorite period of the carious disease, which is one of the main causes of apical periodontitis, but also a period of life where sport is common and it is also a factor in risk of trauma. ${ }^{12,13}$

These results are in phase with the data from the literature. Indeed, a recent epidemiological study of Huumonen showed a strong predominance of men and an average age of 40 years. ${ }^{14}$

Previous studies around the world had found the same trends. ${ }^{15,16}$

Clinically, the majority of patients $(86.3 \%)$ came for consultation for aesthetic and / or functional reasons and only 7 patients $(13.7 \%)$ for a routine visit.

Generally, the most common reason for consultation in endodontics is pain ${ }^{17}$ however, the values recorded in this study are highly understandable because chronic apical periodontitis is the main criterion for inclusion of the study population, are of the chronic line characterized by the absence of clinical symptoms (pain) and generally discovered fortuitously on the X-ray during a consultation for an aesthetic and / or functional reason.

The distribution of the population according to tooth type showed that the upper anterior sector, more particularly the incisors, was the most concerned with a percentage of $68.6 \%(\mathrm{~N}=35)$, followed by molars with $23.5 \%$, and finally the premolars with $7.8 \%$.

This high representability of the incisors is in line with Andréansen's work as they are the most exposed teeth to extrinsic alveolo- dental traumas. ${ }^{13}$

Clinical observation of the different cases in the present study showed only one case (2\%) presenting a sinus tract in the vestibule opposite the causal tooth and three other cases $(5.6 \%)$ presenting a periodontal depths.

A fistula is an exteriorized drainage pathway for apical inflammatory fluids. Although its appearance is often ignored by the patient, the fistula is created as a result of acute episodes primary or secondary: the drainage of the abscess by the fistula causes the disappearance of the phenomena of overpressure and it follows the regression of pain and swelling.

Examination of the mucosa showed an absence of swelling in the great majority of cases $(n=49$ or $96.1 \%$ ) and only 2 cases (3.9\%) presented a slight peripapical swelling. This result has an anatomopathological explanation. In fact, chronic apical periodontitis corresponds to an inflammatory defensive response of the peri apical tissues, in which the proliferative component (granulation tissue) outweighs the exudative component (abscess). The absence of pain is explained by the absence of overpressure due to the peripheral bone reaction.

The distribution of the degree of mobility of the tooth according to the Mühlemann classification showed that the majority of cases $(n=45$, ie $88.2 \%)$ had a physiological mobility, and that few cases showed visible but inferior transversal mobility. to 1 $\mathrm{mm}$ and other greater than $1 \mathrm{~mm}$ with each 3 cases (or $5.9 \%)$.

Chronic apical periodontitis has timid clinical signs that are often inconsistent with the stage, activity, and severity of the etiopathogenic process, so lesion growth may result in clinically detectable versions / migrations or even mobilities. When the lesion is bulky, a hard feeling on the digital palpate can sit in the vestibule. ${ }^{18}$

Radiographic assessment of the periodontal ligament status showed ligament thickening in $82.4 \%$ (42 cases). That of peri apical status showed that scores 5 and 4 of the peri apical index (PAI) are the most recorded with respectively a percentage of $45.1 \%$ and $31.4 \%$. The low scores 3 and 2 are the least encountered in the present study with respectively 15.7 and $7.8 \%$.

This distribution of periapical status is related to the methodology of the study because only teeth with peri-radicular bone lesion (PAI greater than or equal to 2 ) were included. This distribution is interesting for monitoring the radiological evolution of periapical healing dynamics over time after endodontic treatment. Finally, it should be noted that chronic apical periodontitis is a specific pathological entity in the group of endodontic pulp and periodontal diseases.

The existence of a CAP means the systematic presence of a pathway of bacterial contamination of endodontic origin associated with a defensive reaction of the periapical tissues. ${ }^{2}$

To solve the infectious problem, the practitioner must eliminate the endocanalar infection, that is to say perform the endodontic treatment, and this, whatever the stage and the clinical form of the pathology. ${ }^{19,20}$

Because of this, the difficulty of the diagnosis lies not in the final therapeutic decision-making, but rather in the establishment of the differential diagnosis between the clinical forms. This difficulty is increased by the absence of pathognomonic signs ("gold 
standard") and the low level of scientific proof between the anatomopathological data and the clinical pictures characterizing each type of P.A.

The presence of a radio clear lesion of endodontic etiology at the peri-radicular region may suggest three different histological entities: the inflammatory granuloma, the pocket cyst, and the true cyst. Only the first two entities can be resolved by orthograde endodontic treatment. The resolution of the true cyst can then pass only by its complete surgical eviction.

The diagnostic possibility of differentiating the cyst from the granuloma would make it possible to direct the therapy towards the most appropriate management: orthograde or retrograde pathway.

Currently, the differentiation of these clinical entities can be done only by means of histopathological examination after eviction of the lesion appended to the resected apical fragment.

On the basis of these findings, it would be interesting to carry out further studies with a view to finding less invasive diagnostic means than apical surgery followed by pathological examination to determine the histological nature.

Conic beam computed tomography (CBCT) allows a three-dimensional assessment of peri-radicular lesions and may facilitate peri apical cyst screening. Thus studies could attempt to develop or evaluate the predictive validity of a cyst screening method based on volumetric analysis alone of CBCT or associated with specific radiological criteria such as volume, density and radiological characteristics of the cyst lesion.

Other diagnostic means such as contrasting solutions, albumin test, and ultrasound to determine the presence of a cyst constitute relevant lines of research.

\section{Conclusion}

The present study showed that chronic apical periodontitis are more common in men and have modest clinical signs that are often inconsistent; which shows that their diagnosis is mainly radiographic.

\section{References}

1. Toure B, Kane AW, Sarr M, Ngom CT, Boucher Y. Prevalence and technical quality of root fillings in Dakar, Senegal. Int Endod J. 2008;41(1):41-49.

2. Nair PNR. Apical periodontitis : a dynamic encounter between root canal infection and host response. $J$ Periodontol. 1997;13:121-48.

3. Lasfargues JJ. Le diagnostique clinique des parodontites apicales. Réalités cliniques 2001;12(2):149-54.

4. Muhlemann H. Ten years of tooth mobility measurements. J Periodontol. 1960;31:110-22.

5. Ørstavik D, Kerekes K, Eriksen HM. The periapical index: a scoring system for radiographic assessment of apical periodontitis. Endod Dent Traumatol. 1986;2(1):20-34
6. Pockpa ZA, Didia EL, Mobio YS, Coulibaly NT, Djeredou KB. Evaluation of periodontal health of prosthetic abutments. Pilot study about 100 abutments of crowns and bridges. Odontostomatol Trop. 2015;38(152):39-47.

7. 7.Tordai B, Schreindorfer K, Lempel E, Krajczár K. Factors affecting ultrasonic removal of separated endodontic instruments: A retrospective clinical study. Quintessence Int. 2018;49(4):257-66.

8. Colombo M, Bassi C, Beltrami R et al. Radiographic technical quality of root canal treatment performed by a new rotary single-file system. Ann Stomatol (Roma). 2017;8(1):18-22.

9. Perlea P, Nistor CC, Iliescu MG, Iliescu AA. The use of cone beam computer tomography in the diagnosis and management of internal root resorption associated with chronic apical periodontitis: a case report. Rom $J$ Morphol Embryol. 2015;56(1):223-27.

10. Pitcher B, Alaqla A, Noujeim M, Wealleans JA, Kotsakis G, Chrepa V. Decision trees for preoperative periapical cyst screening using cone-beam computed tomography. $J$ Endod. 2017;43(3):383-88.

11. 11.Zengin AZ, Celenk P, Sumer AP, Cankaya S. Evaluation of traumatic dental injuries in a group of Turkish population. Niger J Clin Pract. 2015;18(1):8689.

12. 12.Andreasen FM, Kahler B. Pulpal response after acute dental injury in the permanent dentition: clinical implications-a review. J Endod. 2015;41(3):299-308.

13. Andreasen FM, Kahler B. Diagnosis of acute dental trauma: the importance of standardized documentation: a review. Dent Traumatol. 2015;31(5):340-49.

14. Huumonen S, Suominen AL, Vehkalahti MM. Prevalence of apical periodontitis in root filled teeth:findings from a nationwide survey in Finland. Int Endod $J$ 2017;50(3):229-36.

15. Boucher Y, Matossian L, Rillard F, Machtou P. Radiographie evaluation ofthe prevalence and technical quality of root canal treatment in a french subpopulation. Int Endod J 2002;35:229-38.

16. Jimenez-Pinzon A, Segura-Egea JJ, Poyato-Ferrera M, Velasco-Ortega E, Rios-Santos JV. Prevalence of apical periodontis and frequency of root filled teeth in an adult Spanish population. Int Endod J. 2004;37:167-73.

17. Seck A, Ndiaye D, Niang SO, Kaboré AD, Bane K, LéyeBenoistF, Sarr M, Faye B. Le retraitement endodontique au département d'odontologie de Dakar: étude rétrospective à propos de $72 \mathrm{cas}$. Rev Col OdontoStomatol Afr Chir Maxillo-fac. 2016;23:31-35.

18. Croitoru IC, CrăiȚoiu Ş, Petcu CM, Mihăilescu OA, Pascu RM, Bobic AG, et al. Clinical, imagistic and histopathological study of chronic apical periodontitis. Rom J Morphol Embryol. 2016;57(2):719-28.

19. Martinho FC, Gomes CC, Nascimento GN, Gomes APM, Leite FRM. Clinical comparison of the effectiveness of 7 and 14-day intracanal medications in root canal disinfection and inflammatory cytokines. Clin Oral Investig. 2017;4:123-30.

20. Rodrigues RCV, Zandi H, Kristoffersen AK, Enersen M, Mdala I, Ørstavik D, Rôças IN, Siqueira JF. Influence of the Apical Preparation Size and the Irrigant Type on Bacterial Reduction in Root Canal-treated Teeth with Apical Periodontitis. J Endod. 2017;5:145-50. 